\title{
Reviewers for the Special Issue
}

We would like to thank the following people who reviewed papers submitted for publication in this special issue.

Richard Arvey
Michael Bagby
Beverly Balos
John Bargh
Gene Borgida
John Briere
Angela Browne
Margaret Bull-Kovera
Diana Burgess
Martha Burt
Pam Casey
Caroline Cochran
Bonnie Dansky
Mary Ann Dutton
Joel Dvoskin
Phoebe Ellsworth
Charles Ewing
David Faigman
David Farrington
Barry Feld
Norman Finkel
Diane Follingstad
Lawrence Friedman
Rosemary Gartner
Marti-Hope Gonzales
Jane Goodman-Delahunty
Stephanie Goodwin
Edie Greene
Patricia Griffin
James Gruber

Richard Arvey

Beverly Balos

John Bargh

Gene Borgida

John Briere

Angela Browne

Diana Burgess

Martha Burt

Pam Casey

Caroline Cochran

Bonnie Dansky

Joel Dvoskin

Phoebe Ellsworth

Charles Ewing

David Faigman

David Farrington

Barry Feld

Norman Finkel

Lawrence Friedman

Rosemary Gartner

Marti-Hope Gonzales

Jane Goodman-Delahunty

Edie Greene

James Gruber

\author{
Beth Haney \\ Craig Haney \\ Stephen Hart \\ Jeff Hauggard \\ Kirk Heilbrun \\ Seth Kalichman \\ Stanley Kaplan \\ Florence Kaslow \\ Mary Koss \\ Janet Kottke \\ E. Allen Lind \\ Marsha Liss \\ Henry Martinez \\ James J. McDonald, Jr. \\ J. Reid Meloy \\ Gary Melton \\ Stephen Morse \\ Michael Nietzel \\ Jim Ogloff \\ Stephen Penrod \\ Melissa Polusny \\ Paula Popovich \\ John Pryor \\ Neil Rector \\ N. Dickon Reppucci \\ Heidi Resnick \\ Marnie Rice \\ Stephanie Riger \\ Caton Roberts \\ David Ross
}


Barry Ruback

Laurie Rudman

Daniel Shuman

Mark Small

Donald Songer

Loretta Stalans
Margaret Stockdale

Linda Teplin

Martie Thompson

Tom Tyler

Amye Warren

Cathy Spatz Widom 